\title{
Safety and Efficacy of Elosulfase Alfa in Australian Patients with Morquio A Syndrome: A Phase 3b Study
}

Journal of Inborn Errors of Metabolism \& Screening 2020, Volume 8: e20200001 DOI: 10.1590/2326-4594-JIEMS-2020-0001

\author{
Kaustuv Bhattacharya ${ }^{1,2}$ (D), Shanti Balasubramaniam ${ }^{2,3}$, \\ Kevin Murray ${ }^{3}$, Heidi Peters 4 , David Ketteridge ${ }^{5}$, Anita Inwood 6 , \\ Joy Lee ${ }^{4}$, Carolyn Ellaway ${ }^{1,2}$, Penny Owens ${ }^{1}$, Melanie Wong ${ }^{2,7}$, \\ Christine Ly ${ }^{8}$ and Jim McGill'
}

\begin{abstract}
The safety and efficacy of elosulfase alfa were evaluated in a multicenter, open-label, phase 3b study in Australian Morquio A patients, consisting of a 49-week initial phase and an extension phase until elosulfase alfa was government funded. Thirteen patients (1-27 years) were enrolled. No new safety concerns were identified over 138 weeks. Most drug-related adverse events were mild or moderate in severity; none led to study discontinuation. After 49 weeks of treatment, median improvements from baseline were seen in the 6-minute walk test $(+41.0 \mathrm{~m})$, 3-minute stair climb test $(+14.0 \mathrm{stairs} / \mathrm{min})$, forced vital capacity $(+16.4 \%)$, forced expiratory volume in 1 second $(+14.1 \%)$, urine keratan sulfate $(-7.1 \mu \mathrm{g} / \mathrm{mg}$ creatinine), and pain intensity. Growth, cardiac function, sleep, and quality of life results were mixed or stable. These results provide further evidence of the acceptable safety/tolerability profile of elosulfase alfa. The improvements in endurance, pulmonary function, and pain support findings from previous studies.
\end{abstract}

\section{Keywords:}

efficacy, elosulfase alfa, enzyme replacement therapy, mucopolysaccharidosis IVA, Morquio A syndrome, safety.

ClinicalTrials.gov Identifier: NCT01966029

\section{Introduction}

Morquio A syndrome, or mucopolysaccharidosis (MPS) IVA, is a rare lysosomal storage disorder caused by a deficiency in the enzyme $N$-acetylgalactosamine-6-sulfatase (GALNS; EC 3.1.6.4). GALNS deficiency results in impaired catabolism of the glycosaminoglycans (GAGs) keratan sulfate (KS) and chondroitin-6-sulfate and accumulation of the non-degraded GAGs in tissues and organs, resulting in the characteristic phenotype of MPS IVA[1].

Most patients with Morquio A syndrome appear healthy at birth, but ultimately develop an array of multi-systemic manifestations that worsen with disease progression[2]. Characteristic clinical features of Morquio A syndrome include skeletal dysplasia (dysostosis multiplex, spinal cord compression), disproportionate short stature with short trunk and neck, hyperextensible joints, and several non-skeletal manifestations such as impaired pulmonary function, airway obstruction, cardiac abnormalities, impaired vision (glaucoma, corneal clouding), hearing loss, and hepatomegaly[3,4]. There is

\footnotetext{
1 Sydney Children's Hospitals Network, Genetic Metabolic Disorders Service, Sydney, New South Wales, Australia.

${ }^{2}$ University of Sydney, Faculty of Medicine and Health, Sydney, New South Wales, Australia.

${ }^{3}$ Perth Children's Hospital, Department of Rheumatology and Metabolic Medicine, Perth, Australia.

${ }^{4}$ The Royal Children's Hospital Melbourne, Department of Paediatrics, Melbourne, Victoria, Australia.

${ }^{5}$ Women's and Children's Hospital Adelaide, North Adelaide, South Australia, Australia.

${ }^{6}$ Queensland Children's Hospital, Brisbane, Queensland, Australia.

${ }^{7}$ Children's Hospital at Westmead, Department of Immunology, Sydney, New South Wales, Australia.

${ }^{8}$ BioMarin Pharmaceutical Inc, Novato, California, United States.
}

Received January 16, 2020. Accepted for publication March 02, 2020.

\section{Corresponding Author:}

Kaustuv Bhattacharya, Sydney Children's Hospitals Network, Genetic Metabolic Disorders Service Sydney, New South Wales, Australia.

Email: Kaustuv.bhattacharya@health.nsw.gov.au. 
a broad spectrum of severity[1]. Regardless of severity, Morquio A syndrome is associated with an increased risk of morbidity and a reduced lifespan, with the main cause of death being respiratory failure[5]. Tracheal obstruction is a frequently occurring manifestation of Morquio A syndrome that may lead to life-threatening complications[6,7].

Enzyme replacement therapy (ERT) with elosulfase alfa, a genetically engineered form of human GALNS, is infused intravenously weekly to help correct the enzyme deficiency and restore cell function[8]. A number of clinical trials have provided evidence for the efficacy and safety of this treatment [8-13]. The pivotal double-blind, placebo-controlled, phase 3 study of elosulfase alfa (MOR-004[9]), including 176 patients $\geq 5$ years of age, and its long-term extension (MOR-005[10]) showed a statistically significant improvement in the distance walked in the 6-minute walk test (6MWT), a rapid and sustained reduction in urine KS (uKS), as well as sustained numerical improvements in pulmonary function measures, and activities of daily living (ADL) after 120 weeks of treatment $[10,11,14]$. The safety profile of elosulfase alfa was considered acceptable, with most drugrelated adverse events (AEs) being mild or moderate infusionassociated reactions (IARs), which were generally manageable with slowing of the infusion rate and symptomatic and/or prophylactic anaphylaxis-suppressing medication $[9,10]$. The natural history of Morquio A syndrome is progression of the disease and deterioration of endurance, pulmonary function, and the ability to perform ADL over time. Therefore, the long-term maintenance of these measures shows the benefit of elosulfase alfa on patients with Morquio A syndrome[10,11,14].

Here, we present the results of an Australian study that evaluated the safety and efficacy of elosulfase alfa in patients with Morquio A syndrome.

\section{Materials and Methods}

\section{Study design and objectives}

A multicenter, open-label phase $3 \mathrm{~b}$ study was performed in patients with Morquio A syndrome from five Australian centers (\#NCT01966029). The study consisted of a 49-week initial treatment phase, followed by a long-term extension phase until elosulfase alfa was approved for government funding through the Life Saving Drugs Program. The primary objective of the initial phase was to evaluate the safety and tolerability of elosulfase alfa administration in Australian patients. The primary objective of the extension phase was to provide patients enrolled in the initial phase access to elosulfase alfa until the drug was funded in Australia and to continue to assess longterm safety. Secondary objectives of the study were to assess the efficacy of elosulfase alfa with respect to endurance, pulmonary function, growth velocity, uKS levels, cardiac function, sleep, quality of life, and pain.

Patients with Morquio A syndrome, confirmed by a documented GALNS deficiency, received weekly infusions of elosulfase alfa, $2.0 \mathrm{mg} / \mathrm{kg}$. During the 49 -week initial phase of the study, the study sites completed age-appropriate assessments of drug efficacy at 0,25 and 49 weeks. Patients completing the initial phase were then enrolled in the extension phase. During the extension phase, only safety assessments were required with efficacy assessments performed according to each institution's standard of care.

Study drug infusions were administered intravenously following the study drug information guidelines. Specifically, the infusion was started at a low rate that was gradually increased every 15 minutes for 1 hour, then hourly, as tolerated by patients, until a maximum rate was reached. The entire volume was administered over approximately 4 hours.

The study was conducted in compliance with the Code of Ethics of the World Medical Association (Declaration of Helsinki), and approved by each institution's Ethics Committee. All patients or their parents provided written, signed informed consent to participate in the study.

\section{Safety evaluation}

The safety analysis included all patients who received at least one infusion of elosulfase alfa.

Safety variables included AEs, routine physical examination (including standard neurological examination), clinical laboratory tests (serum chemistry, hematology, and urinalysis), vital signs, pulse oximetry, 12-lead electrocardiogram (ECG), documentation of prior and concomitant medications (including analgesic medication use), immunogenicity tests, pregnancy test (if appropriate), and cervical spine imaging. AEs were graded according to the National Cancer Institute Common Terminology Criteria for Adverse Events v. 4.0. Assessments and procedures for safety evaluations are outlined further in Supplementary file 1. Blood samples for total antibody (TAb) and neutralizing antibody $(\mathrm{NAb})$ testing using the ImmunoCAP ${ }^{\mathrm{TM}}$ 1000 Assay System (Thermofisher Scientific), an automated sandwich immunoassay system, were collected at baseline, week 25, and week 49 . NAb were not measured if TAb was negative. Samples for total IgE were collected at baseline and stored for testing. In patients who experienced a severe IAR, an IAR requiring cessation of infusion, anaphylaxis or a serious hypersensitivity event shortly after cessation of the infusion, the baseline total IgE sample was analyzed and additional blood samples for total IgE and drug-specific IgE testing were drawn and run.

\section{Efficacy evaluation}

All enrolled patients with at least one efficacy measurement after treatment initiation were included in the efficacy analysis. Ageappropriate efficacy assessments included endurance as measured by the $6 \mathrm{MWT}$ and 3-minute stair climb test (3MSCT), pulmonary function tests (including forced expiratory volume in 1 second $\left[\mathrm{FEV}_{1}\right]$ and forced vital capacity $[\mathrm{FVC}]$, forced inspiratory vital capacity [FIVC], and maximum voluntary ventilation [MVV]), 
uKS normalized to creatinine, anthropometric measurements (standing height, sitting height, length, knee height [as clinically indicated], head circumference, and weight), quality of life (QoL) as assessed using the Pediatric Quality of Life Inventory (PedsQL) or Short Form 36 (SF-36, for adults), and pain as assessed by the Adolescent Pediatric Pain Tool (APPT). Sleep apnea was assessed (if applicable) as a tertiary outcome.

The PedsQL is a 23-item questionnaire appropriate for children and adolescents $<18$ years consisting of four multidimensional domains: physical, emotional, social, and school functioning[15]. Items are reverse-scored and linearly transformed to a 0-100 scale with higher scores indicating better health-related QoL. The SF-36 has been developed for use in adults and adolescents $\geq 16$ years and assesses QoL in two subdomains, i.e. the Physical Component Summary and the Mental Component Summary[16]. The APPT is a validated, multidimensional tool developed to evaluate pain in children and adolescents of 8-17 years old[17]. It consists of a body outline to identify pain locations, the WordGraphic Rating Scale (WGRS), which is a $10-\mathrm{cm}$ visual analog scale with five equidistant pain intensity word anchors (no pain, little pain, medium pain, large pain, worst possible pain), and a pain quality word descriptor list.

For patients $<7$ years of age, QoL and pain assessments were completed by caregivers of the patients provided they were appropriately based on the health status and developmental maturity of the individual patient. Assessments and procedures for efficacy are further outlined in Supplementary file 1.

\section{Statistical analysis}

All safety data were summarized descriptively. All AEs were coded using the Medical Dictionary for Regulatory Activities. The incidence of AEs was summarized by System Organ Class (SOC), Preferred Term (PT), relationship to study drug, and severity (https://www.meddra.org/). Serious adverse events (SAEs), deaths, AEs leading to study drug discontinuation, and study withdrawals were reported. All AE summaries included only treatment-emergent AEs reported during the study period (AEs that occur after the first study drug infusion). IARs were summarized by SOC, PT, and severity.

All efficacy variables were summarized descriptively using means, standard deviations (SDs), standard errors, medians, minimum-maximum ranges, interquartile ranges, and $95 \%$ confidence intervals (CI). Dosing compliance was calculated as a percentage of total planned dose.

\section{Results}

\section{Patient characteristics}

Thirteen patients with confirmed Morquio A syndrome, comprising five females and eight males, were enrolled in the study. Table 1 summarizes demographics and baseline characteristics of the study population. Mean $( \pm S D)$ age was
Table 1. Patient demographics and baseline characteristics.

\begin{tabular}{|c|c|}
\hline Demographic / baseline variable & $\mathbf{N}=13$ \\
\hline \multicolumn{2}{|l|}{ Age at enrolment, years } \\
\hline Mean $( \pm \mathrm{SD})$ & $9.3( \pm 7.7)$ \\
\hline Median & 5.0 \\
\hline Min-max & $1-27$ \\
\hline$<5$ years of age, $N(\%)$ & $4(30.8)$ \\
\hline \multicolumn{2}{|l|}{ Gender } \\
\hline Female, N (\%) & $5(38.5)$ \\
\hline Male, N (\%) & $8(61.5)$ \\
\hline \multicolumn{2}{|l|}{ Race } \\
\hline Asian, N (\%) & $1(7.7 \%)$ \\
\hline Caucasian, N (\%) & $9(69.2 \%)$ \\
\hline Other, N (\%) & $3(23.1 \%)$ \\
\hline \multicolumn{2}{|l|}{ Age at diagnosis, years } \\
\hline Mean $( \pm \mathrm{SD})$ & $2.2( \pm 1.4)$ \\
\hline Min-max & $0-5$ \\
\hline \multicolumn{2}{|l|}{ uKS, $\mu \mathrm{g} / \mathrm{mg}$ creatinine } \\
\hline Mean $( \pm S D)$ & $47.7( \pm 33.4)$ \\
\hline Min-max & $8-111$ \\
\hline \multicolumn{2}{|l|}{ Standing height, cm } \\
\hline Mean $( \pm S D)$ & $94.5( \pm 6.2)$ \\
\hline Median & 94.3 \\
\hline Min-max & $87-108$ \\
\hline Standing height < 3rd percentile, N (\%) & $12(92.3)$ \\
\hline
\end{tabular}

SD: standard deviation; uKS: urine keratan sulfate.

$9.3( \pm 7.7)$ years and ranged from 1 to 27 years. Most patients (69.2\%) were Caucasian; no patients identifying as Aboriginal / Torres Strait Islander were included. Standing height was below the third percentile in 12 of 13 patients (92.3\%).

\section{Safety}

Patients were generally compliant to study drug administrations throughout the study. Median dosing compliance was 97.6 (range 87-99) \%. Patients had a median of 1 (range 0-4) incomplete infusions of a median total number of 132 (range 99-152) infusions.

The study did not identify new safety concerns. All patients experienced at least one AE; 12 out of 13 (92.3\%) patients had at least one drug-related $\mathrm{AE}$, but no patients discontinued the study drug due to a drug-related AE (Table 2). Two patients had instances of infusion interruption due to IARs that were managed by medical intervention (Supplementary file 2). One patient discontinued the study at week 104 due to an SAE: spinal cord infarction unrelated to the study drug. The patient experienced worsening respiratory symptoms and was identified as having tracheal stenosis in addition to cervical canal stenosis and instability. Following surgery to correct these, the patient 
Table 2. Summary of safety results

\begin{tabular}{lc}
\hline & Number of subjects (\%) \\
\hline Any SAE & $9(69.2 \%)$ \\
$\mathbf{1}$ drug-related AE & $12(92.3 \%)$ \\
Grade 1 & $4(30.8 \%)$ \\
Grade 2 & $5(38.5 \%)$ \\
Grade 3 & $2(15.4 \%)$ \\
Grade 4 & $1(7.7 \%)$ \\
Leading to study drug discontinuation & a \\
SAE & $0(0.0 \%)$ \\
Death & $1(7.7 \%)$ \\
\hline
\end{tabular}

AE: adverse event; SAE: serious adverse event

ane subject discontinued the study at week 104 for the primary reason of an AE (spinal cord infarction). Treatment for this patient was considered completed because the patient discontinued the study due to a medical reason per study protocol

experienced a spinal cord infarction. The SAE was not felt to be related to the study drug. The most frequently reported study drug-related AEs were diarrhea (53.8\% of patients), vomiting (53.8\%), pyrexia (46.2\%), nasopharyngitis (38.5\%), and pain in extremity (38.5\%) (Supplementary file 3). All patients experienced IARs. The investigators reported the majority of study drugrelated AEs as either mild or moderate. Three drug-related AEs were reported as severe. These consisted of two separate episodes of dyspnea for the same patient reported as probably drug-related and managed with oxygen. The third AE, an event of incarcerated umbilical hernia, is likely a disease manifestation erroneously reported as possibly drug-related.

Nine (69.2\%) patients had at least one SAE, which were most frequently reported as MPS-related clinical manifestations such as knee deformity (23.1\%) and sleep apnea syndrome (23.1\%). These events were not related to the study drug, but occur with disease progression. Only one patient experienced a SAE possibly related to the study drug (grade 4 respiratory failure) 3 days after the last infusion. This patient had a history of asthma and breathlessness that may have contributed to the event. The event of respiratory failure resolved after dexamethasone treatment. No action was taken related to administration of elosulfase alfa after the event. One individual aged 6 years at recruitment developed significant IARs (Supplementary file 2). No deaths occurred in the study. No drug-specific IgE positive results were reported during the study. Twelve patients (92.3\%) developed NAbs, but no correlations were detected between higher TAb titers or NAb positivity with either endurance measures or with the incidence of hypersensitivity AEs.

\section{Efficacy}

Efficacy data are presented for the initial phase of the study only, due to the limited number of efficacy assessments during the extension phase.
Endurance tests (6MWT and 3MSCT) were performed in patients $\geq 5$ years $(\mathrm{N}=9)$. Two patients participating in the endurance tests used walking aids (walker or walking frame). Two out of nine patients $\geq 5$ years old (aged 21 and 27 years) were physically unable to perform the 3MSCT at baseline. At baseline, patients had a median 6MWT distance of 286 (range 154-383) $\mathrm{m}$ and a median of 39 (range 28-63) stairs/min in the 3MSCT. Median change from baseline in the $6 \mathrm{MWT}$ was $+33.5(95 \% \mathrm{CI}-14.3,65.9) \mathrm{m}$ at 25 weeks $(\mathrm{N}=9)$ and $+41.0(95 \%$ CI $3.3,107.8) \mathrm{m}$ at 49 weeks $(\mathrm{N}=8)$; median percent changes from baseline were $+11.2 \%$ and $+13.9 \%$ at 25 and 49 weeks, respectively. Median change from baseline in the 3MSCT was $+3.3(95 \%$ CI $4.6,18.4)$ stairs/min at 25 weeks $(\mathrm{N}=7)$ and +14.0 $(95 \%$ CI $0.6,22.1)$ stairs/min at 49 weeks $(\mathrm{N}=6)$; median percent changes from baseline were $+9.1 \%$ and $+31.7 \%$ at 25 and 49 weeks, respectively. Overall, younger patients (5-11 years) had better outcomes in the 6MWT and 3MSCT than older patients (15-27 years) (Figure 1).

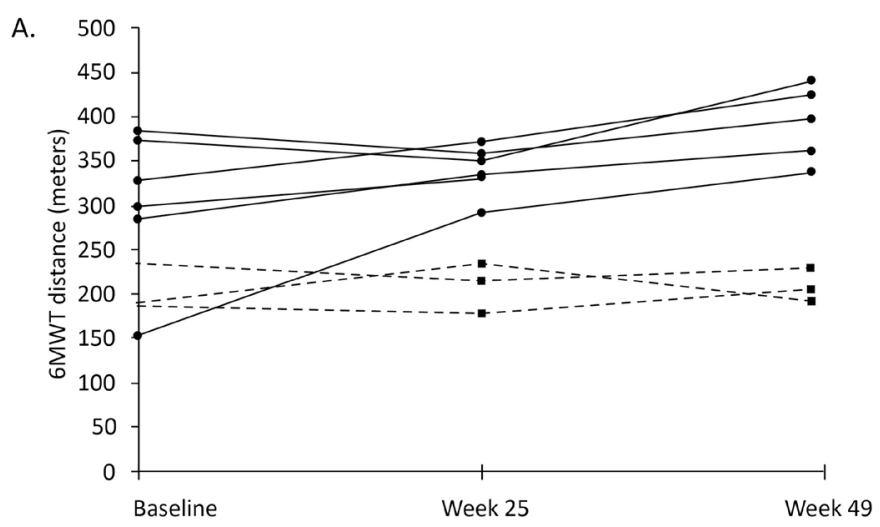

B.

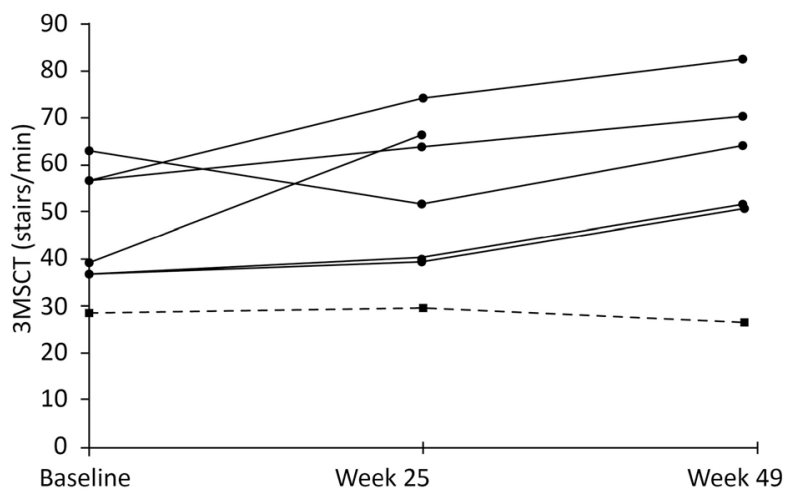

Figure 1. Endurance test results from individual patients: $(A)$ 6-minute walk test (6MWT; $\mathrm{N}=9$ ) and (B) 3-minute stair climb test (3MSCT; N=7); outcomes in children (5-11 years; solid lines) and adults (15-27 years, dashed lines).

Pulmonary function tests, assessed in patients $>7$ years of age $(\mathrm{N}=6)$, showed improvements in $\mathrm{FVC}$ and $\mathrm{FEV}_{1}$ (Figure 2). 


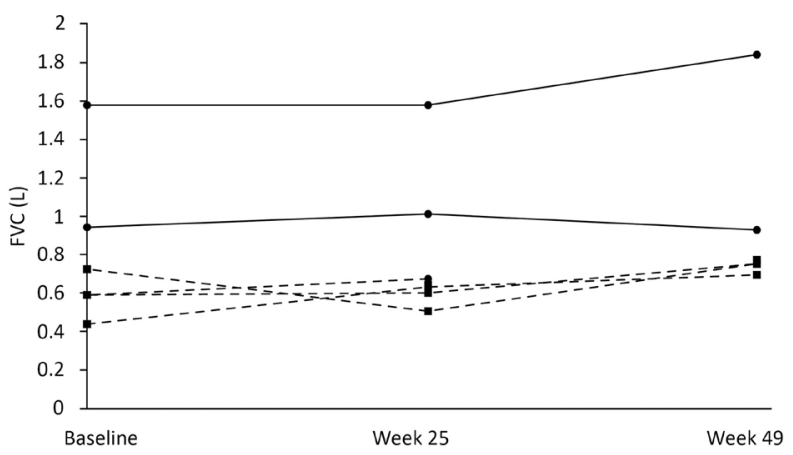

B.

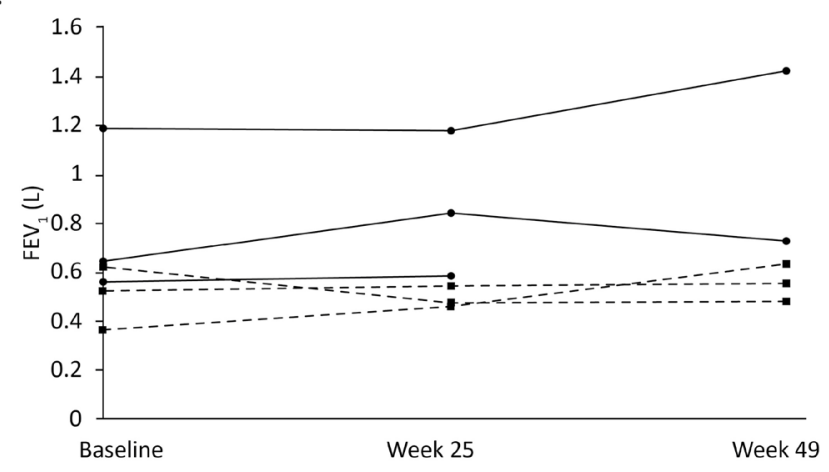

Figure 2. Individual pulmonary function data $(N=6)$ : $(A)$ Forced vital capacity ( $F V C)$ and $(B)$ forced expiratory volume in 1 second $\left(\mathrm{FEV}_{1}\right)$ in children (5-11 years; solid lines) and adults (15-27 years, dashed lines).

Median FVC was 0.7 (range $0.4,1.6$ ) $\mathrm{L}$ at baseline; median baseline $\mathrm{FEV}_{1}$ was 0.6 (range $0.4,1.2$ ) L. Median change in FVC was $0.0 \mathrm{~L}(95 \% \mathrm{CI}-0.1,0.2)$ and $+0.1(95 \% \mathrm{CI} 0.0,0.3) \mathrm{L}$ at 25 weeks and 49 weeks, respectively. Median change in $\mathrm{FEV}_{1}$ was $0.0(95 \% \mathrm{CI}-0.1,0.1) \mathrm{L}$ and $+0.1(95 \% \mathrm{CI} 0.0,0.2) \mathrm{L}$ at 25 weeks and 49 weeks, respectively. Median percent change in FVC was $+5.4 \%$ and $+16.4 \%$ at 25 weeks and 49 weeks, respectively. Median percent change in $\mathrm{FEV}_{1}$ was $+3.7 \%$ and $+14.1 \%$ at 25 weeks and 49 weeks, respectively. No change over time was seen in FIVC. No conclusions could be made for MVV due to very limited data (available for 2 patients at 49 weeks) and as the degree of effort applied for this test can impact outcome measurement. Individual patient data for $\mathrm{FVC}$ and $\mathrm{FEV}_{1}$ show varying results between patients, though improvements were seen in both children and adults (Figure 2). It should be noted that one of the patients had cystic fibrosis in addition to Morquio A syndrome, but had stable respiratory function during the study.

For all patients, median baseline uKS was 33.7 (range 8-111) $\mu \mathrm{g} / \mathrm{mg}$ creatinine. The median change in uKS was $-11.1 \mu \mathrm{g} /$ $\mathrm{mg}$ creatinine after 25 weeks and $-7.1 \mu \mathrm{g} / \mathrm{mg}$ creatinine after 49 weeks. The median percent change was $32.6 \%$ at week 25 and 30.6 at week 49 . Individual patient data are provided in Supplementary file 4.
Parent-reported QoL data from the PedsQL were available for 11 patients; patient-reported data for seven patients. At baseline, both parent- and patient-reported median scores were low (indicating poor health-related QoL) and tended to be lowest at older ages. On a 0-100 scale, with higher scores indicating better health-related QoL, parent-reported baseline Total Scores ranged from 40.5 to 100; patient-reported baseline Total Scores ranged from 41.3 to 65.2 (Supplementary file 5). Parent-reported baseline Physical Functioning Scores ranged from 19 to 100; patient-reported baseline Physical Functioning Scores ranged from 19 to 66. As different versions of the PedsQL had to be used for different age categories, patient numbers within each analysis were too limited to allow for any conclusions regarding mean changes in scores over time. Individual patient data did not show consistent results (Supplementary file 5). Only two patients completed the SF-36 assessment. No conclusions could be drawn from these assessments.

Pain data from the APPT were available for 10 patients. Median baseline WGRS score was 5.0 at baseline $(\mathrm{N}=10)$ and decreased (i.e. improved) by a median of 0.3 points at 25 weeks $(\mathrm{N}=10)$ and 1.5 points at 49 weeks $(\mathrm{N}=9)$. The lower extremities and the back were the most commonly reported body site locations for pain.

Mean standing height, sitting height, weight, and length increased from baseline over time.

Standing height increased by a median of $1.6 \%$ after 25 weeks ( $\mathrm{N}=11)$ and $4.5 \%$ after 49 weeks $(\mathrm{N}=10)$. However, median normalized height (z-score) at baseline was -4.3 and remained relatively unchanged after 25 weeks and 49 weeks. Supplementary file 6 presents individual longitudinal height data from patients $<12$ years of age at baseline $(\mathrm{N}=10)$, showing increases in height in all patients, and overall larger increases in the youngest patients. Height z-score remained unchanged in these patients.

At baseline, 4/11 patients (36.4\%) had abnormal ECGs, including sinus arrhythmia/tachycardia, incomplete right bundle branch block, and nodal rhythm. The number of patients with ECG abnormalities remained generally unchanged throughout the study. Two patients with a normal baseline ECG had an abnormal ECG at weeks 25 and 49 (sinus bradycardia with first degree atrioventricular block, and sinus arrhythmia, respectively). One patient with nodal rhythm on ECG at baseline had a normal ECG at weeks 25 and 49 . Holter monitoring in this patient performed at week 25 demonstrated the nodal rhythm, which resolved by 49 weeks.

Abnormal sleep was observed in $8 / 11$ (72.7\%) patients at baseline, 4/8 (50.0\%) patients at week 25, and 8/10 (80.0\%) patients at week 49 or later. One patient with abnormal sleep at baseline had normal sleep at week 49; two patients with normal sleep at baseline had abnormal sleep at 49 weeks.

\section{Discussion}

Morquio A syndrome is a debilitating disease associated with several skeletal and non-skeletal clinical manifestations, 
J. inborn errors metab. screen.

with a natural history of progressive deterioration in endurance, cardiorespiratory function, mobility, and QoL $[2,18]$. Elosulfase alfa is currently the only approved pharmacological treatment for Morquio A syndrome that targets the biological cause of the disease, i.e. deficiency of the GAG-degrading lysosomal enzyme, GALNS. Evidence supporting the use of hematopoietic stem cell transplantation (HSCT) in patients with Morquio A syndrome is limited, and this treatment approach is currently not recommended[19]. In the pivotal phase 3 clinical trial (MOR-004/005), Morquio A patients treated with elosulfase alfa for up to 120 weeks showed a significant improvement in the 6MWT, a rapid and sustained reduction in uKS, and numerical improvements in the 3MSCT, pulmonary function, and the ability to perform ADL[9-11,14]. Other studies suggested favorable effects of elosulfase alfa on exercise capacity, pain, and muscle strength in patients with relatively good baseline endurance and improvements in growth in patients starting treatment before the age of 5 years $[12,20,21]$. The MOR-004/005 study was conducted in 17 countries in Northern and Latin America, Europe, and Asia. The goal of the present study was to provide further evidence for the safety and efficacy of elosulfase alfa in Australian patients with Morquio A syndrome. Elosulfase alfa has been approved in Australia for the treatment of Morquio A syndrome since December 2014 (www.tga.gov.au). Overall, the safety and efficacy results of the Australian study support findings from the pivotal phase 3 study and other clinical trials with elosulfase alfa.

The study did not identify new safety signals of elosulfase alfa after a mean total exposure period of over 2.5 years. However, one individual had severe IARs that required intensive management for a period of time whilst on the study, which was withdrawn during the extension phase. The most frequently reported drugrelated AEs (diarrhea, vomiting, pyrexia, nasopharyngitis, and pain in extremity) were mostly mild or moderate in severity and similar to those reported in previous studies[12,13]. Although most patients experienced SAEs, these were predominantly MPS-related symptoms such as knee deformity and sleep apnea, not related to the study drug. Only one patient experienced a SAE possibly related to treatment (respiratory failure), and one temporarily required infusion of ERT over 24 hours after a severe IAR. Most patients developed antibodies against elosulfase alfa, but these did not seem to affect improvements in endurance measures (6MWT, 3MSCT) or the incidence of hypersensitivity AEs. This finding is in line with the immunogenicity results from the pivotal MOR-004 study[9].

Baseline assessments showed abnormalities in uKS, endurance, height, and QoL in the study population. Median uKS level was 33.7 (reference range 5-20[22]) $\mu \mathrm{g} / \mathrm{mg}$ creatinine. Baseline standing height was below the third percentile in all patients but one; median height $\mathrm{z}$-score was -4.3. It should be noted that 6MWT distance is dependent on height $[23,24]$ and that the tallest study participant was only $108 \mathrm{~cm}$. The median baseline 6MWT distance $(286 \mathrm{~m})$ was below the $10^{\text {th }}$ percentile reported for 4-year old children with a mean height of $107 \mathrm{~cm}(322$ $\mathrm{m})$. No 6MWT reference data are available for younger (smaller) children. QoL, as assessed with the PedsQL, was generally poor. With the exception of one patient, scores were considerably below PedsQL Total Scores of 80-84 and Physical Functioning Scores of 82-88 reported for a healthy population[15]. Median pain intensity (ATTP WGRS score) was 5.0 at baseline, which indicates medium pain, and is comparable with pain scores previously reported for Morquio A patients[12,25]. The small stature, poor endurance, and low QoL scores of most patients suggest that the majority of patients in the study have a classical phenotype.

Efficacy outcomes at 25 and 49 weeks were generally in accordance with published data. Median uKS levels decreased with treatment, confirming previous findings $[10,12,13,21]$. However, uKS levels at baseline and effects of treatment were highly variable between patients, likely due to differences in phenotypes and ages at treatment onset. Although a reduction in uKS can show a pharmacodynamic response to treatment, the clinical significance of this marker remains to be confirmed[26,27]. uKS passes via the kidney and studies have shown that it does not contain all forms of undegraded KS that are present in the blood and tissues[26]. Although alternative biomarkers have recently been suggested (e.g. mono-sulfated and di-sulfated blood KS and pro-inflammatory factors), their relevance as surrogate markers for therapeutic efficacy has not yet been established[26,27]. Endurance results in the 6MWT and 3MSCT were comparable with those reported for the MOR$004 / 005$ study $[9,10]$. Median increases from baseline in the $6 \mathrm{MWT}$ were $33.5 \mathrm{~m}$ at 25 weeks and $41.0 \mathrm{~m}$ at 49 weeks, versus mean increases ranging between $30.7 \mathrm{~m}$ and $42.2 \mathrm{~m}$ after 24 to 120 weeks reported for patients continuously treated with elosulfase alfa $2.0 \mathrm{mg} / \mathrm{kg} /$ week (QW-QW cohort) in MOR-004/005[10]. The median improvement from baseline in the 3MSCT of 14.0 stairs/min after 49 weeks was higher than the mean changes seen after 24 to 120 weeks of treatment in the MOR-004/005 QW-QW cohort (ranging between 4.6 and 5.9 stairs $/ \mathrm{min}$ )[10]. These 6MWT and 3MSCT results should be interpreted in light of the natural history of Morquio A syndrome, which is associated with a gradual decline in endurance over time[10,28]. The better endurance results versus the MOR004/005 study might be due to the younger age of the patients included in the Australian study and less severe endurance impairments at baseline. The latter is supported by the finding that younger patients had better endurance outcomes than older patients (Figure 1). It should also be noted that two out of nine patients $\geq 5$ years were not included in the 3MSCT analysis as they were physically unable to perform the test at baseline. The pulmonary function measures FVC and $\mathrm{FEV}_{1}$ also increased during the course of the study, with median increases of around $16 \%$ and $14 \%$ after 49 weeks of treatment. These increases are greater than the 7-8\% after 120 weeks of treatment reported for the MOR-004/005 study[11], probably related to the younger age of the Australian study population. As a comparison, natural history data previously showed changes in FVC and $\mathrm{FEV}_{1}$ of $2.2 \%$ and $0.9 \%$, respectively, after 1 year 
and of $2.6 \%$ and $-0.6 \%$, respectively, after 2 years in untreated patients. As lung volume is related to body size, growth likely played an important role in the pulmonary function outcomes in the Australian study population.

In this Australian study, standing height increased by a median of $4.5 \%$ after 49 weeks of treatment. Normalized height (z-score) remained constant, suggesting normal growth during the study period. However, because of the limited number of patients in the study, the relatively short follow-up time, and lack of historical growth data from these patients, no conclusions could be made regarding the impact of treatment on growth. It should be noted that a constant height $z$-score does not exclude a treatment effect, considering the progressive nature of the disease. The pivotal MOR-004 study showed non-statistically significant improvements versus placebo in normalized standing height $(+0.1)$ and growth rate $\mathrm{z}$-scores $(+0.4)$ after 24 weeks of treatment in males $\leq 18$ years and females $\leq 15$ years[8]. Longer follow-up data are needed to further assess the impact of ERT on growth in Morquio A patients.

Pain evaluation in the ATTP showed a reduction in median pain intensity from 5.0 at baseline to 3.5 after 49 weeks, confirming published findings[12,20]. No conclusions could be made regarding changes in QoL over time, as different types of questionnaires were used to assess QoL in different age categories, resulting in a very limited number of patients completing each questionnaire. Cardiac and sleep results were generally stable over time, which may be due to the relatively short follow-up period. Most of the reported ECG abnormalities, including sinus arrhythmia/tachycardia and right bundle branch block have been described previously for Morquio A patients[2,29]. One patient developed sinus bradycardia with first degree atrioventricular block during the study. To our knowledge this manifestation has not been reported before in this patient population. Sleep abnormalities in Morquio A patients can be due to tracheal narrowing, causing progressive sleep apnea[6]. The long-term impact of ERT on cardiac abnormalities and sleep disorders in Morquio A patients remains to be established. Skeletal dysplasia was not assessed during the study, so no conclusions can be made regarding the impact of treatment on this measure. To date, there is no evidence from clinical trials that ERT has an impact on the progression of bone disease in Morquio A patients.

A limitation of the current study is its open-label design, which does not allow for a direct comparison of efficacy outcomes with untreated Morquio A patients. It cannot be excluded that the observed effects are partly due to a better follow-up of patients or a motivating or training effect (in case of the endurance tests). Nevertheless, data from the Morquio A Clinical Assessment Program natural history study (MorCAP [2]) suggest that patients would have had less favorable outcomes without treatment. Overall, patients from MorCAP showed a gradual decline in endurance in the 6MWT and 3MSCT over time and a reduction in pulmonary function measures in patients $>14$ years of age $[10,11,30]$. In younger patients, increases in FVC and FEV1 were limited $(<10 \%)[11,30]$.
Other limitations of this study include the small number of patients in the study, and missing data for many of the efficacy assessments. In part, the missing data are due to the fact that not all patients were physically able or considered old enough to perform or complete the assessments. This may have led to the exclusion of patients with poorer physical abilities from the efficacy analysis.

\section{Conclusions}

The results of this Australian phase $3 \mathrm{~b}$ study provide further evidence for the acceptable safety and tolerability profile of elosulfase alfa in patients with Morquio A syndrome. The study confirmed the safety of elosulfase alfa that has been reported in previous studies. The individual patient data presented here provide better insight into how elosulfase alfa can impact on patients' health in different ways. Despite the progressive nature of the disease, positive effects were observed in endurance, pulmonary function, and pain after 1 year of treatment, confirming findings from previous clinical trials. Further studies are warranted to evaluate the impact of elosulfase alfa on other variables such as growth, cardiac function, and QoL in the long term.

\section{Acknowledgements}

The authors are grateful to Ismar Healthcare NV for their assistance in the writing of this manuscript, which was funded by BioMarin Pharmaceutical Inc., Novato, CA, USA, and to the staff of the Pain and Anaesthesia Research Centre at the Royal Adelaide Hospital for their contribution to the study.

\section{Funding acknowledgement}

This work was supported by BioMarin Pharmaceutical, Inc.

\section{Declaration of Conflicting Interests}

Kaustuv Bhattacharya reports travel grants, honoraria, and research funding from BioMarin Pharmaceutical Australia Pty Ltd.

Shanti Balasubramaniam reports travel grants and honoraria from BioMarin and research funding from BioMarin to the Princess Margaret Hospital for conducting the clinical study discussed in this publication.

Kevin Murray reports clinical trial research funding from BioMarin Pharmaceutical Australia Pty Ltd, for the purpose of this study to Princess Margaret Hospital (Perth Children's Hospital).

Heidi Peters reports travel grants, honoraria and clinical trial research funding from BioMarin Pharmaceutical Australia Pty Ltd, outside the submitted work.

David Ketteridge reports travel grants, honoraria and research funding from BioMarin Pharmaceutical Australia Pty Ltd, 
travel fees and honoraria from Genzyme, research funding from Amicus, and salary from SA Health, outside the submitted work. Anita Inwood is a member of the BioMarin Australian PKU Advisory Board, has received travel grants from BioMarin and is an investigator for the MARS Registry.

Joy Lee reports travel grants and clinical trial research funding from BioMarin Pharmaceutical Australia Pty Ltd, outside the submitted work.

Carolyn Ellaway has conducted speaker, consultancy, medical advisory board work and received travel grants from BioMarin, Sanofi Genzyme and Shire.

Penny Owens reports payments from BioMarin to act as Trial Coordinator in the phase $3 \mathrm{~b}$ study.

Melanie Wong has nothing to disclose.

Christine Ly is a former employee of BioMarin Pharmaceutical Inc.

Jim McGill has conducted speaker, consultancy, board member work and received travel grants from Actelion, BioMarin Pharmaceutical Inc., Sanofi Genzyme and Shire. He has been an investigator for BioMarin Pharmaceutical Inc.

\section{Data sharing statement}

The de-identified individual participant data that underlie the results reported in this article (including text, tables, figures, and appendices) will be made available together with the research protocol and data dictionaries, for non-commercial, academic purposes. Additional supporting documents may be available upon request. Investigators will be able to request access to these data and supporting documents via a website (www. BioMarin.com) beginning 6 months and ending 2 years after publication. Data associated with any ongoing development program will be made available within 6 months after approval of relevant product. Requests must include a research proposal clarifying how the data will be used, including proposed analysis methodology. Research proposals will be evaluated relative to publically available criteria available at www.BioMarin.com to determine if access will be given, contingent upon execution of a data access agreement with BioMarin Pharmaceutical Inc.

\section{Supplementary material}

The following online material is available for this article: Supplementary file 1 - Schedule of safety and efficacy assessments. Supplementary file 2 - Details on infusion associated reactions (IAR).

Supplementary file 3 - Frequently reported drug-related adverse events by Preferred Term.

Supplementary file 4 - Individual normalized urine keratan sulfate data (uKS) at baseline and at 25 weeks and 49 weeks follow-up.

Supplementary file 5 - Parent- and patient-reported PedsQL Total and Physical function scores at baseline, week 25, and week 49 by patient.
Supplementary file 6 - Individual standing height data from patients below 12 years (yrs) of age at baseline.

\section{References}

1. Hendriksz CJ, Harmatz P, Beck M, et al. Review of clinical presentation and diagnosis of mucopolysaccharidosis IVA. Mol Genet Metab. 2013;110(1-2):54-64. doi:10.1016/j. ymgme.2013.04.002

2. Harmatz P, Mengel KE, Giugliani R, et al. The Morquio A Clinical Assessment Program: baseline results illustrating progressive, multisystemic clinical impairments in Morquio A subjects. Mol Genet Metab. 2013;109(1):5461. doi:10.1016/j.ymgme.2013.01.021

3. Hendriksz CJ, Giugliani R, Harmatz P, et al. Design, baseline characteristics, and early findings of the MPS VI (mucopolysaccharidosis VI) Clinical Surveillance Program (CSP). J Inherit Metab Dis. 2013;36(2):373-384. doi:10.1007/ s10545-011-9410-9

4. Hendriksz CJ, Al-Jawad M, Berger KI, et al. Clinical overview and treatment options for non-skeletal manifestations of mucopolysaccharidosis type IVA. J Inherit Metab Dis. 2013;36(2):309-322. doi:10.1007/s10545-012-9459-0

5. Lavery C, Hendriksz C. Mortality in Patients with Morquio Syndrome A. JIMD Rep. 2015;15:59-66.

6. Tomatsu S, Averill LW, Sawamoto K, et al. Obstructive airway in Morquio A syndrome, the past, the present and the future. Mol Genet Metab. 2016;117(2):150-156. doi:10.1016/j.ymgme.2015.09.007

7. Pizarro C, Davies RR, Theroux M, Spurrier EA, Averill LW, Tomatsu S. Surgical reconstruction for severe tracheal obstruction in Morquio A syndrome. Ann Thorac Surg. 2016;102(4):e329-e331. doi:10.1016/j. athoracsur.2016.02.113

8. Hendriksz CJ, Giugliani R, Harmatz P, et al. Multi-domain impact of elosufase alfa in Morquio A syndrome in the pivotal phase III trial. Mol Genet Metab. 2015;114(2):178185. doi:10.1016/j.ymgme.2014.08.012

9. Hendriksz CJ, Burton B, Fleming TR, et al. Efficacy and safety of enzyme replacement therapy with BMN 110 (elosulfase alfa) for Morquio A syndrome (mucopolysaccharidosis IVA): a phase 3 randomised placebo-controlled study. J Inherit Metab Dis. 2014;37(6):979-990. doi:10.1007/ s10545-014-9715-6

10. Hendriksz CJ, Parini R, AlSayed MD, et al. Long-term endurance and safety of elosulfase alfa enzyme replacement therapy in patients with Morquio A syndrome. $\mathrm{Mol}$ Genet Metab. 2016;119(1-2):131-143. doi:10.1016/j. ymgme.2016.05.018

11. Hendriksz CJ, Berger KI, Parini R, et al. Impact of longterm elosulfase alfa treatment on respiratory function in patients with Morquio A syndrome. J Inherit Metab Dis. 2016;39(6):839-847. doi:10.1007/s10545-016-9973-6 
12. Burton BK, Berger KI, Lewis GD, et al. Safety and physiological effects of two different doses of elosulfase alfa in patients with Morquio A syndrome: A randomized, doubleblind, pilot study. Am J Med Genet A. 2015;167A(10):22722281. doi:10.1002/ajmg.a.37172

13. Harmatz PR, Mengel E, Geberhiwot T, et al. Impact of elosulfase alfa in patients with morquio A syndrome who have limited ambulation: an open-label, phase 2 study. Am J Med Genet A. 2016;173(2):375-383. doi:10.1002/ ajmg.a.38014

14. Hendriksz CJ, Parini R, AlSayed M, et al. Impact of longterm elosulfase alfa on activities of daily living in patients with Morquio A syndrome in an open-label, multi-center, phase 3 extension study. Mol Genet Metab. 2018;123:127134. doi:10.1016/j.ymgme.2017.11.015

15. Varni JW, Burwinkle TM. The PedsQL ${ }^{\mathrm{TM}}$ as a patientreported outcome in children and adolescents with attention-deficit/hyperactivity disorder: a populationbased study. Health Qual Life Outcomes. 2006;4(1):26. doi:10.1186/1477-7525-4-26

16. RAND Health Care. 36-Item Short Form Survey Instrument (SF-36). Accessed April 25, 2019. https://www.rand.org/ health-care/surveys_tools/mos/36-item-short-form/ survey-instrument.html

17. Jacob E, Mack AK, Savedra M, Van Cleve L, Wilkie DJ. Adolescent pediatric pain tool for multidimensional measurement of pain in children and adolescents. Pain Manag Nurs. 2014;15(3):694-706. doi:10.1016/j. pmn.2013.03.002

18. Hendriksz CJ, Al-Jawad M, Berger KI, et al. Clinical overview and treatment options for non-skeletal manifestations of mucopolysaccharidosis type IVA. J Inherit Metab Dis. 2013;36(2):309-322. doi:10.1007/s10545-012-9459-0

19. Akyol MU, Alden TD, Amartino H, et al. Recommendations for the management of MPS IVA: systematic evidenceand consensus-based guidance. Orphanet J Rare Dis. 2019;14:137. doi:10.1186/s13023-019-1074-9

20. Treadwell M, Harmatz PR, Burton BK, et al. Impact of elosulfase alfa pain in patients with Morquio A Syndrome over 52 weeks: MOR-008: a randomized, double-blind, pilot study. J Inborn Errors Metab Screen. 2017;5:1-12. doi:10.1177/2326409817718850
21. Jones SA, Bialer M, Parini R, et al. Safety and clinical activity of elosulfase alfa in pediatric patients with Morquio A syndrome (mucopolysaccharidosis IVA) less than 5 years. Pediatr Res. 2015;78(6):717-722. doi:10.1038/pr.2015.169

22. Wood T, Bodamer OA, Burin MG, et al. Expert recommendations for the laboratory diagnosis of MPS VI. Mol Genet Metab. 2012;106(1):73-82. doi:10.1016/j. ymgme.2012.02.005

23. Lammers AE, Hislop AA, Flynn Y, Haworth SG. The 6-minute walk test: normal values for children of 4-11 years of age. Arch Dis Child. 2008;93(6):464-468. doi:10.1136/ adc. 2007.123653

24. Li AM, Yin J, Au JT, et al. Standard reference for the sixminute-walk test in healthy children aged 7 to 16 years. Am J Respir Crit Care Med. 2007;176(2):174-180. doi:10.1164/ rccm.200607-883OC

25. Hendriksz CJ, Lavery C, Coker M, et al. Burden of disease in patients with Morquio A syndrome: results from an international patient-reported outcomes survey. Orphanet J Rare Dis. 2014;9:32. doi:10.1186/1750-1172-9-32

26. Khan SA, Mason RW, Giugliani R, et al. Glycosaminoglycans analysis in blood and urine of patients with mucopolysaccharidosis. Mol Genet Metab. 2018;125(12):44-52. doi:10.1016/j.ymgme.2018.04.011

27. Fujitsuka H, Sawamoto K, Peracha H, et al. Biomarkers in patients with mucopolysaccharidosis type II and IV. Mol Genet Metab Rep. 2019;19:100455. doi:10.1016/j. ymgmr.2019.100455

28. Kenth JJ, Thompson G, Fullwood C, Wilkinson S, Jones S, Bruce IA. The characterisation of pulmonary function in patients with mucopolysaccharidoses IVA: A longitudinal analysis. Mol Genet Metab Rep. 2019;20:100487. doi:10.1016/j.ymgmr.2019.100487

29. Kampmann C, Abu-Tair T, Gökce S, et al. Heart and cardiovascular involvement in patients with mucopolysaccharidosis type IVA (Morquio-A Syndrome). PLoS One. 2016;11(9):e0162612. doi:10.1371/journal. pone.0162612

30. Harmatz PR, Mengel KE, Giugliani R, et al. Longitudinal analysis of endurance and respiratory function from a natural history study of Morquio A syndrome. $\mathrm{Mol}$ Genet Metab. 2015;114(2):186-194. doi:10.1016/j. ymgme.2014.10.015 